\title{
Effects of functional endoscopic sinus surgery on the acoustics of the sinonasal tract*
}

\author{
Miriam Havel' ', Sven Becker², Maria Schuster' ${ }^{1}$, Thorsten Johnson³, \\ Rhinology 55: 81-89, 2017 \\ Andreas Maier ${ }^{4}$, Johan Sundberg ${ }^{5}$ \\ https://doi.org/10.4193/Rhino16.229 \\ ' Dept. of Otorhinolaryngology, Head\&Neck Surgery, Section Phoniatrics, Munich University Hospital, Munich, Germany \\ *Received for publication: \\ 2 Dept. of Otorhinolaryngology, Head\&Neck Surgery, University Medical Center of Johannes Gutenberg University, Mainz, Germany July 30, 2016 \\ ${ }^{3}$ Dept. of Radiology, Munich University Hospital, Munich, Germany \\ Accepted: November 11, 2016 \\ ${ }^{4}$ Dept. of Computer Science, Friedrich-Alexander-University, Erlangen, Germany \\ ${ }^{5}$ Dept. of Speech, Music and Hearing, School of Computer Science and Communication, KTH (Royal Institute of Technology), \\ Stockholm, Sweden
}

\begin{abstract}
Background: Nasal and paranasal cavities are supposed to contribute substantially to the vocal tract resonator properties. However, their acoustical effects as well as the effects of sinus surgery on the voice remain unclear. In this work we investigate resonance phenomena of paranasal sinuses prior to and after various rhinosurgical procedures in cadaveric human sinonasal tracts and corresponding 3D casts.
\end{abstract}

Methodology: Nasal and paranasal cavities of formalin-preserved cadavers and corresponding 3D replicas were excited by sinetone sweeps from an earphone placed in the epipharynx. The response was picked up by a microphone at the nostrils. Different FESS procedures were performed and the acoustical responses following excitation were recorded. The measured acoustical changes in the obtained transfer functions were then evaluated.

Results: Marked low frequency dips were detected in the transfer functions when sinus cavities were included in the nasal resonator system. These dips showed a significant correlation with sinus volumes. Following FESS procedures they moved upwards in frequency depending on the extent of the surgical intervention.

Conclusions: The transfer functions obtained in cadaveric situs and 3D replicas showed dips at the resonance frequencies of the paranasal cavities. Marked acoustic effects in terms of increase in dip frequency following FESS procedures were reproducibly documented.

Key words: functional endoscopic sinus surgery, paranasal sinuses, resonance frequency, sinonasal tract, cadaveric study, 3D replica

\section{Introduction}

Acoustic properties of the nasal and paranasal cavities represent a long-standing issue in voice research. Some authors claim that the nose and the air-filled paranasal sinuses in the facial cranium contribute substantial acoustic effects to the human voice.

Particularly, comparisons of spectra from physical or computer simulated models of the nasal tract and in vivo recorded nasals and nasalized vowels have shown effects expected from shunting cavities ${ }^{(1-4)}$. Other authors found no relation between voice quality and the paranasal sinuses; these are regarded as poor resonators due to the limited size of ostia, turbinates covering the ostia and sinonasal mucosa which should dampen vibration ${ }^{(5-7)}$. Also from a clinical point of view, voice alteration following sinus surgery seems to be a controversial issue; some authors have failed to observe voice effects after rhinosurgical interventions ${ }^{(8,}$ 9). On the other hand, others have found measurable acoustical changes ${ }^{(10-12)}$. 


\section{Overview of experimental acoustical approaches}

The detailed anatomy of the nasal and paranasal cavities is quite complex and shows high interindividual variability as well as a marked left/right asymmetry. This variable morphology produces an extremely complex frequency response obtained in acoustical experiments, differing both within and between individuals ${ }^{(4,13-15)}$. Additionally, the sinonasal tract including the sinus ostia region is lined with mucosa with considerable swelling potential due to variety of exogenous factors as well as the endogenous, ultradian controlled nasal cycle ${ }^{(16)}$. This non-controllable variation in patency of the sinus ostia and nasal cavity can be expected to substantially alter the acoustical properties of the nasal tract. Further, the velopharyngeal opening, which is highly variable itself, substantially determines the degree of coupling between the nasal and vocal tracts and thus affects the acoustical result ${ }^{(17)}$.

To rule out the contribution of paranasal cavities to the nasal sound, attempts have been made to investigate the resonance properties of the nose by application of decongestants and thus by direct comparison of normal and decongested condition ${ }^{(2,18)}$. However, as the inferior turbinate represents the primarily decongestant-sensitive component of the nasal cavity, the observed acoustical differences may have predominantly emanated from the volume reduction of this structure.

Another approach consisted in blocking the sinus ostia by Proetz method (flooding the sinuses with iodine-containing contrast agent under $\mathrm{x}$-ray examination) or by gauze bandage stuffing ${ }^{(19)}$. However, the mechanical irritation caused by these procedures may have provided considerable alteration of endonasal mucosal condition with subsequent uncontrollable changes of the acoustic properties.

Effects of sinus surgery: a brief overview of clinical research Clinical investigations of voice alteration in patients undergoing FESS, mostly for treatment of rhinosinusitis, have aimed at preand postoperative comparison of various parameters. Some of these investigations have observed perceptual alterations as well as some spectral effects on the voice ${ }^{(10-12)}$. However, Tepper et al. and Ozbal Koc et al. did not identify any acoustic pre-/postoperative alterations in their examined voice recordings ${ }^{(8,9)}$. Using a spectral analysis approach, also Behrmann et al. ${ }^{(20)}$ and Hosemann et al. ${ }^{(11)}$ observed some changes in postoperatively measured acoustical features. However, the patient group in the first study included individuals who underwent various, partly multiple surgical interventions of the upper airway, including rhinosurgical and oropharyngeal procedures, i.e. tonsillectomy and uvulopalatopharyngoplasty ${ }^{(20)}$. Due to the scarce consistency of the results in the latter trial the authors concluded that the highly complex acoustic components of the nasal resonance might not be sufficiently assessed by means of the acoustic measurements used in heterogeneous groups of patients ${ }^{(11)}$.
Avoiding the complexity of spectral analysis, Soneghet et al. made use of nasometry technique to examine the changes in the so-called nasalance score, defined as the quotient of radiated nasal and oral acoustic energy. The significant increase in nasalance score observed on postoperative follow-up was then compared to the normative nasalance values in German speaking population. However, the acoustical contribution of the nasal and paranasal cavities in particular was not distinguished, nor were the basic acoustical features of the observed changes discussed (21).

The relation between nasalance scores and rhinometrically determined nasal patency has been examined in healthy individuals as well as in patients undergoing sinus surgery comparing nasal condition prior to and after decongestant application (22) and FESS intervention, respectively ${ }^{(23,24)}$. Significant changes in both measured parameters were revealed in all trials. However, the two parameters failed to correlate, indicating that the increase of nasalance score were not necessarily associated with increase of nasal patency.

Summarizing, clinical studies on voice quality changes following sinonasal surgery published so far have arrived at controversial conclusions. Different and not directly comparable acoustic measurements and parameters have been applied. The data acquisition and averaging across groups of patients treated for various pathologies by means of different surgical techniques contain several confounding factors that prevent comparability and specification of the potential acoustic effects.

As endonasal surgical procedures on nasal and paranasal cavities rank among the most commonly performed surgical interventions in otorhinolaryngology, it is relevant to further elucidate their potential acoustic impact on speech and voice quality. In the present study we systematically examine acoustic effects of FESS in stable anatomical condition of cadaveric sinonasal tracts and corresponding 3D casts.

\section{Materials and methods}

The present investigation includes four parts, 1) measurement of resonance properties of the nasal tract in cadavers, (a) with the ostia of the maxillary and the sphenoidal sinuses occluded and (b) after removing the occlusion of each of these ostia, one at a time. 2) measurement of resonance properties of the nasal tract in cadavers before and after surgical interventions, 3) measurement of resonance properties of the nasal tract in $3 \mathrm{D}$ replicas based on CT scan imaging of sinonasal tracts, and 4) comparisons of measured and theoretically calculated resonance frequencies of theses models. Details about these measurements will be given below.

The resonance properties were analysed by feeding a sine sweep from an earphone (Jabra BT 3030, Copenhagen, Denmark) mounted in one end of the resonator and picking up the res- 

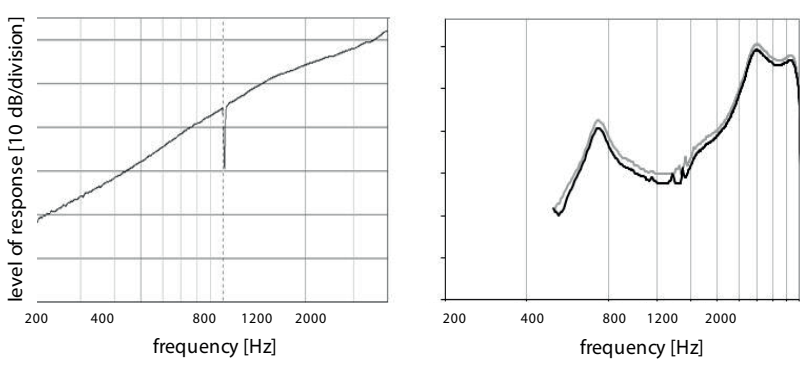

Figure 1. Ex situ earphone to microphone transfer function (left). The frequency of $1000 \mathrm{~Hz}$ is marked with a short interruption dip. Two response curves obtained from sweep tone excitation of isolated nasal cavity (all ostia occluded) in cadaveric situs (right). One of the curves has been shifted by $2 \mathrm{~dB}$, for the sake of demonstration. For closer display of low frequency region a logarithmic frequency scale is used.

ponse in the opposite end by means of an electret microphone (TCM141, AV-Jefe, Taiwan). The sine sweep, range 200 - 4000 Hz, duration $18 \mathrm{~s}$, constant speed along a logarithmic frequency scale, was obtained from the custom made Tombstone ${ }^{\circledR}$ software which simultaneously recorded the response at $16000 \mathrm{~Hz} \mathrm{sam}$ pling frequency (Svante Granqvist, KTH, Stockholm, Sweden). The sound transfer characteristics of the earphone and microphone combination was measured by multiple ex situ recordings in free air using an earphone-microphone distance of $3 \mathrm{~cm}$, approximately, so as to eliminate effects of room resonances. The left panel of Figure 1 shows a typical example of a response curve.

The reproducibility of response curves for the nasal tracts was tested by repeated recordings in cadaveric situs as well as in 3D nasal tract replicas. The right panel of Figure 1 compares a typical result in terms of two response curves obtained from a under identical conditions (both middle meatus and both sphenoidal ostia occluded) in one of the formalin-preserved cadaveric sinonasal tracts (right). The discrepancy between the two in situ recordings was less than $1.5 \mathrm{~dB}$.

Fundamental frequency and sound level of the recorded responses were measured via the Soundswell ${ }^{\oplus}$-software (Saven Hitech AB, Täby, Sweden) using the Corr and Extract subroutines of the Soundswell ${ }^{\circledR}$ work station. Five hundred sample points of these signals, equally spaced in time, were obtained by means of Extract and imported into Excel. This allowed plotting of level as function of frequency. Level curves were corrected according to the frequency response of the earphone and microphone as recorded in free air.

\section{Measurements of cadaveric sinonasal tracts}

The resonance properties of sinonasal tracts were measured in eight formalin-preserved human male cadaveric sinonasal tracts (buffered formalin solution, $40 \mathrm{~g} / \mathrm{l}$ ). The earphone was then placed and hermetically sealed by means of plasticine in the epipharynx. The signal was picked up $2 \mathrm{~cm}$ in front of the nost- rils. The recordings were conducted in a sound treated room. On endoscopic exploration using $0^{\circ}$ and $45^{\circ}$ rigid $4.0 \mathrm{~mm}$ endoscopes (Karl Storz, Tuttlingen, Germany) no pronounced septal deviations were identified in any of the cadavers, and the middle meatus, sphenoidal ostia as well as formalin-preserved mucosa showed normal anatomy as visualized and documented by means of a TelePack X-documentation device (Karl Storz, Germany).

The Institutional Review Board at the University of Munich approved the trial.

The resonance properties of the nasal and sinus cavities were measured in six conditions, prior to (A) and after (B) surgical FESS interventions, performed according to the Simmen \& Jones classification of surgical procedures (25). For each condition, the response to the sine-sweep excitation was measured twice.

A 1. After occlusion of both middle meatus and both sphenoidal ostia (baseline condition, nasal cavity in isolation, i.e. without sinus cavities)

A 2. After subsequent removal of the occlusion of each sinus cavity one by one

B 1. After infundibulotomy and exposure of maxillary ostium

B 2. After widening of maxillary and sphenoidal ostium in terms of maxillary sinusotomy I and sphenoidal sinusotomy I

B 3. After further widening of the ostia (maxillary sinusotomy II and sphenoidal sinusotomy II) including the opening of frontal sinus (frontal sinusotomy II) in terms of frontosphenoethmoidectomy

B 4. After temporary occlusion and removal for every surgically altered ostium, respectively

For condition A1, which was used as reference, both middle meatus and sphenoidal ostia of the cadaveric situs were occluded by endoscopically controlled placement of a viscous mass prepared of maltodextrin food thickener (Nutilis ${ }^{\circledR}$ powder, Nutricia, Germany) and water (for detailed description of the occlusion method see Havel et al. ${ }^{(26)}$ ). For condition A2, the maltodextrin occlusion of the middle meatus/sphenoidal ostium was removed on one side by targeted suction and then re-occluded after sine sweep recording. In this the acoustical contribution of each of the sinus cavities was analysed separately. For conditions B1, B2, B3 and B4 FESS procedures of various extents (varying from infundibulotomy to frontosphenoethmoidectomy) were conducted with subsequent temporary occlusion of the surgically widened sinus ostia. This allowed comparison of acoustical effects of the original and the surgically altered situs. The FESS procedures were conducted in four of the eight cadaveric sinonasal tracts examined after completion of recordings in the preoperative situs. All these rhinosurgical procedures were performed by a surgically trained otolaryngologist (SB) according to the principles of FESS ${ }^{(27)}$, who was blinded towards the aim of the study. All surgeries were standard procedures 


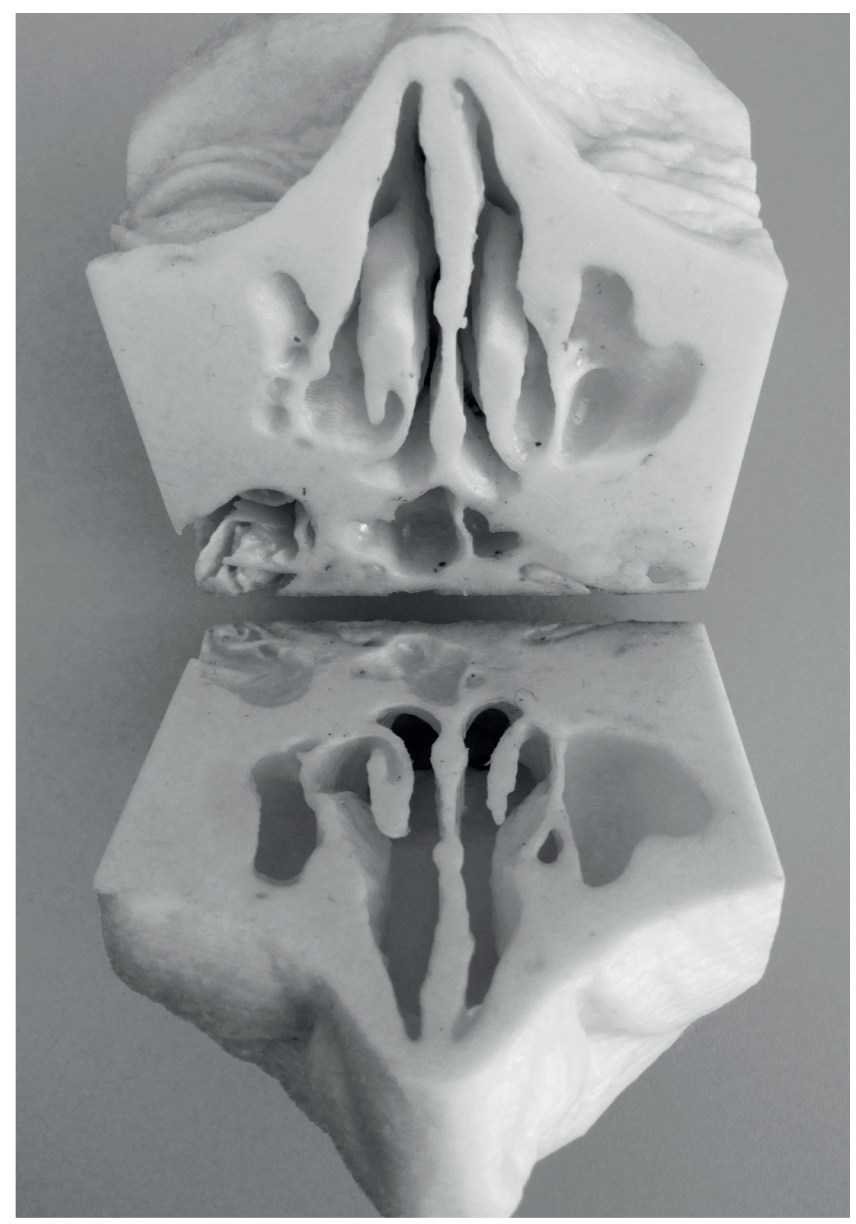

Figure 2. 3D replica of a human sinonasal tract: to enable occlusion of the sinus ostia, the casts were printed axially divided in two halves.

established in the clinical practise for operative treatment of chronic rhinosinusitis. The infundibulotomy, exposing the natural maxillary ostium, was followed by maxillary sinusotomy I and sphenoidal sinusotomy I, as routinely performed in moderate pathologic involvement of these cavities. The final surgical steps was further widening of the maxillary and sphenoidal ostia by maxillary sinusotomy II and sphenoidal sinusotomy II and opening of the frontal recess via ethmoidal cells (frontosphenoethmoidectomy), as recommended for extensive sinonasal pathologies (28).

\section{CT scan imaging and 3D modeling}

High resolution CT scans (slice thickness $0.5 \mathrm{~mm}$, axial acquisition, soft tissue windowing) of the sinonasal tracts were obtained preoperatively as well as after every surgical intervention, thus allowing for volumetric measurements and comparison of structural changes in the dimensions of sinus ostia. Additionally, the DICOM data sets were further processed using ImageJ (Version 1.48, National Institutes of Health, Bethesda, Maryland, 2004) and ITKsnap-software (Version 3.2.0. University of Pennsylvania, Philadelphia, 2004) and the STL files gained served for
$3 \mathrm{D}$ modeling of the sinonasal tracts. The production of the 3D replicas was conducted via FDM (Fused Deposition Modeling) technology using a thermoplast-filament (Stratasys ABS-P430 Dimension Elite, Stratasys, MN, USA). To enable occlusion of the sinus ostia in the replica, the casts were printed axially divided in two halves and the corresponding surfaces of the casts were treated with vaseline to ensure sufficient leak tightness (Figure 2). Eventually, recordings obtained in cadaveric situs with formalin-preserved mucosa and those obtained in thermoplastic 3D replicas in preoperative condition as well as after various FESS procedures were compared.

\section{Calculation of resonance frequencies}

The CT scan data provided were used for volumetric measurements of the examined sinus cavities via ImageJ software. After conversion to binary mode and brightness/contrast adjustments the cavity volume and ostia dimensions were determined using the wand-tracing and straight tool of the ImageJ software in sagittal and coronal reconstructions. The resulting cavity volumes showed a variation of $0.53 \%$ between the reconstruction planes. The measured cavity volume, the cross-sectional area and the length of the ostium were applied in the Helmholtz equation to calculate the resonance frequencies of the given sinus. In the calculation, a tubular ostium configuration was assumed. For calculating the resonance frequency of a given sinus the Helmholtz equation was used:

$$
f=\frac{c}{2 \pi} \sqrt{\frac{A H}{V_{0} \cdot I H}}
$$

where $\mathrm{c}$ is the sound velocity $(34,000 \mathrm{~cm} / \mathrm{s})$

$\mathrm{AH}$ the cross-sectional area of the ostium

$V$ the sinus cavity volume

$\mathrm{IH}$ the effective neck/ostium length (set to $0.2 \mathrm{~cm}$ ).

\section{Statistics}

Statistical analysis was conducted using StatGraphics Centurion XVI (Version 16.1.18, StatPoint Technologies, Warrenton, VI, USA). A student's t-test and Wilcoxon signed-rank test were performed for comparison between data samples and for preoppostop comparison of dip frequency data, respectively. Correlations of the linear regression models were determined via ANOVA. A $p$ value of $<0.05$ was considered statistically significant.

\section{Results}

\section{Baseline condition}

In total, 32 sinus cavities (cadaveric and replica sinuses) were acoustically excited, 85 recordings were eligible for acoustical analysis. 


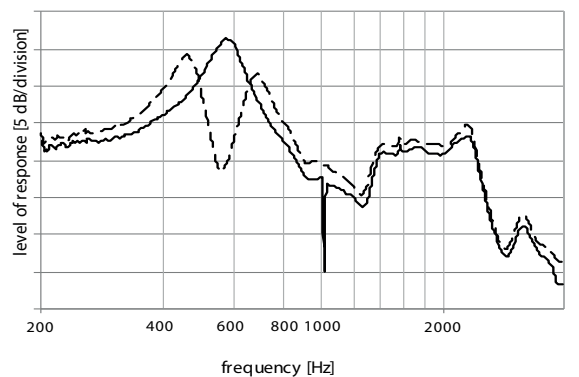

Figure 3. Response to sinesweep excitation of isolated cadaveric nasal cavity with both middle meatus and sphenoidal ostia occluded and after removing the occlusion of the sphenoidal ostium (solid and dashed curves, respectively). The software used for recording the response introduces a marker at $1000 \mathrm{~Hz}$.
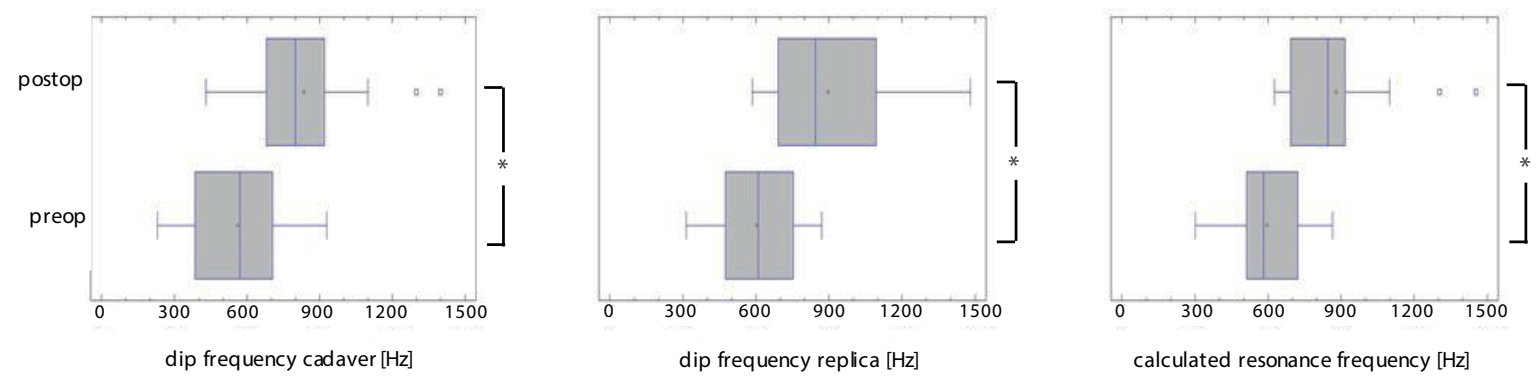

calculated resonance frequency $[\mathrm{Hz}]$
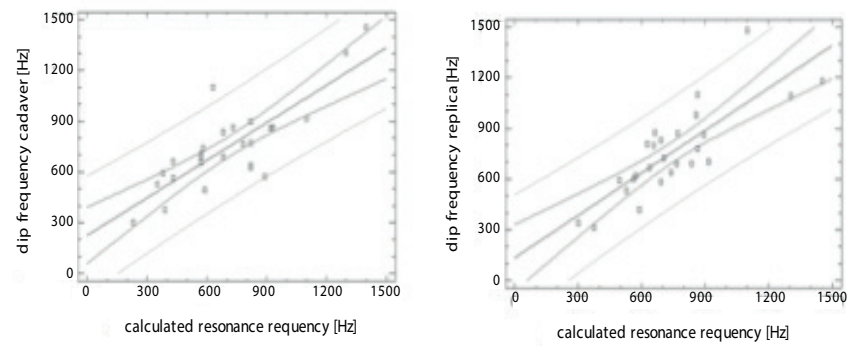

Figure 4. Sinus induced dip frequencies measured after acoustic excitation of cadaveric (left) and 3D replica (right) sinonasal tracts and calculated resonance frequencies, respectively ( $p>0.001$, respectively).

Figure 5. Sinus induced dip frequencies (average \pm standard deviation) prior to (lower „preop" panel, respectively) and following surgical procedures (upper "postop“ panel, respectively) in cadaveric sinonasal tract (left) and 3D replica (middle) as well as the calculated resonance frequencies (right). Surgical widening of the sinus ostia led to an increase in dip frequencies. Significant differences are indicated with asterisk $(*)$.

Considering the acoustical characteristic of the nasal cavity in isolation (i.e. without sinus cavities) as the baseline condition, the occlusion of one of the ostia (middle meatus, sphenoidal ostium, surgically exposed and widened maxillary and sphenoidal ostium, respectively) was subsequently removed, thus showing the acoustical effects of every single sinus separately. A typical acoustical effect of occlusion removal is shown in Figure 3.

In 93\% (79/85) of the exposed ostia a marked dip was detected in the transfer function following removal of the occlusion. In six cases (four middle meatus and two sphenoidal ostia) no effect on the acoustical response was observed, all in the preoperative condition.

The average dip frequency for exposed maxillary ostia amounted to $430 \mathrm{~Hz} \pm 122 \mathrm{~Hz}(\min 230 \mathrm{~Hz}$, $\max 600 \mathrm{~Hz}$ ) and for sphenoidal ostia to $733 \mathrm{~Hz} \pm 133 \mathrm{~Hz}(\min 570 \mathrm{~Hz}$, $\max 930 \mathrm{~Hz})$.

\section{Measured vs. calculated dip frequencies}

The dip frequencies measured experimentally during the acoustical excitation of the cadaveric and replica sinonasal tracts were shown to correlate significantly with the mathematically via Helmholtz-equation calculated dip frequencies using volumetric data derived from CT scan imaging (Figure 4). 

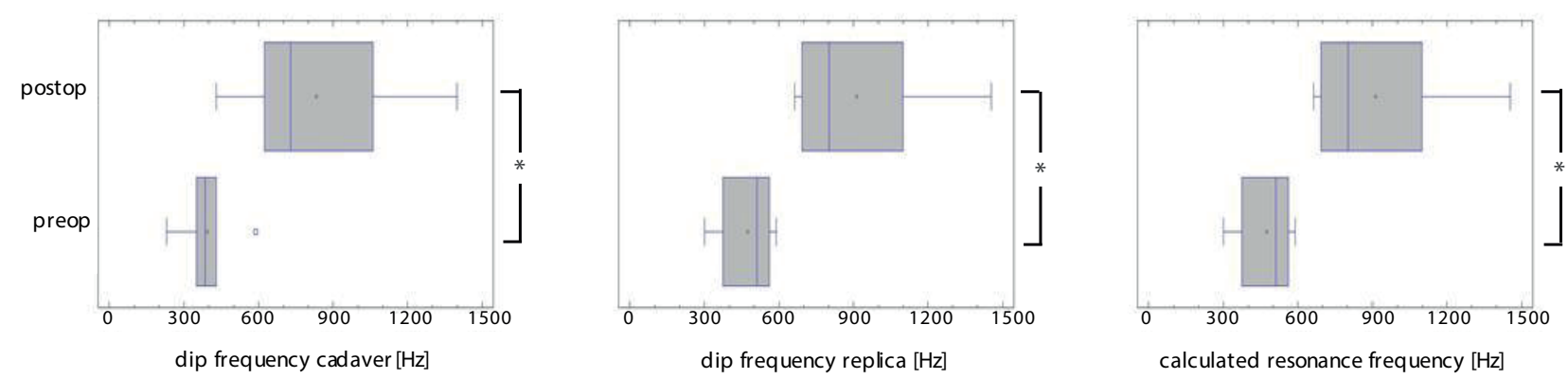

Figure 6. Dip frequencies (average \pm std) of the maxillary sinuses in the preoperative (lower blots) vs. postoperative (upper blots) condition are displayed as measured in the cadaveric situs and 3D cast (left and middle panel) as well as calculated resonances (right). Significant differences are indicated with asterisk $(*)$.
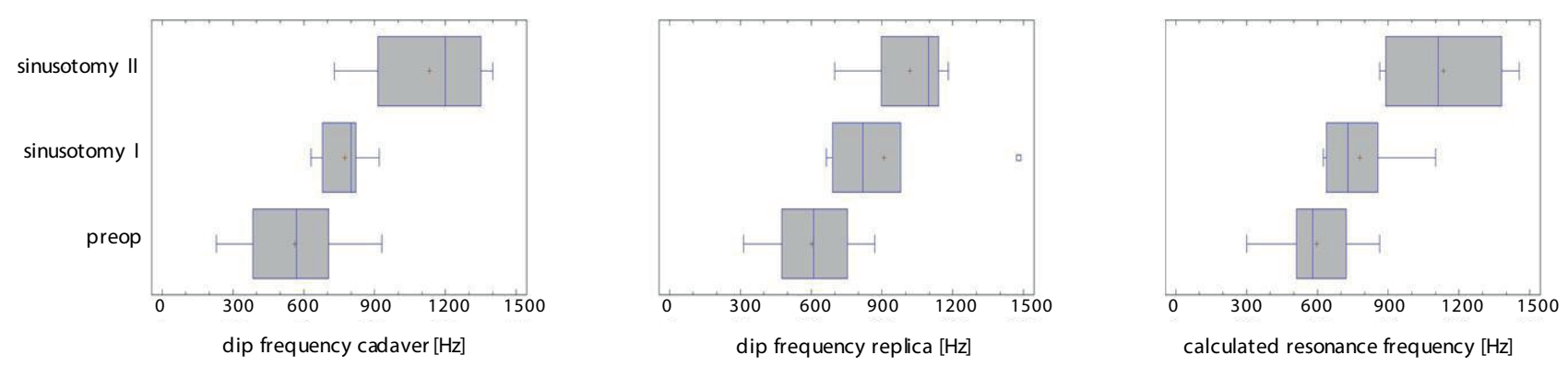

Figure 7. Comparison of dip frequencies (average \pm std) between preoperative condition ("preop", lower blots) and subsequent FESS procedures ("sinusotomy I", middle blots; "sinusotomy II" upper blots) in cadaveric situs (left), 3D cast (middle) as well as calculated resonances (right).

sinus $(p=0.280$ cadaver; $p=0.129$ replica; $p=0.134$ calculation).

Acoustical effects of the subsequent surgical interventions The surgical interventions performed were compared with respect to change of the dip frequency in the transfer function. A comparison between the preoperative condition and the maxillary/sphenoidal sinusotomy I as well as preoperative condition vs. maxillary/sphenoidal sinusotomy II/frontosphenoethmoidectomy procedure showed significant increase in the measured dip frequencies, respectively. Moreover, the particular surgical steps (from maxillary/sphenoidal sinusotomy I towards maxillary/sphenoidal sinusotomy II/frontosphenoethmoidectomy) also showed significant increase in measured dip frequencies. These effects were also observed in the calculated resonance frequencies (Figure 7).

In consequence of the frontosphenoethmoidectomy procedure, the exposure of the frontal recess alongside with the frontal sinuses resulted in marked antiresonances in the measured transfer functions. These frontal sinus induced dips appeared in the range between $1200-1600 \mathrm{~Hz}$ following acoustical excitation of the cadaveric situs as well as of the 3D cast. Statistical evaluation was not considered due to small sample size $(n=2)$.

Dip frequency and sinus cavity volume Regarding the dimension of the sinus cavity, a significant cor- relation between the dip frequency and the cavity volume in the preoperative condition was shown for cadaver and 3D replica respectively ( $p=0.018$, cadaver, $p=0.002$, replica). The calculated resonance frequencies were also found to correlate with the cavity volume $(p=0.002)$.

Postoperatively, correlation between sinus volume and measured dip frequency in the transfer function or calculated resonance frequency did not longer persist $(p=0.210$ in cadaver, $p=0.198$ in replica, $p=0.421$, calculation), showing that the Helmholtz equation did not apply in these surgically altered situs.

\section{Discussion}

In the present work we used an ex vivo experimental setting, applying well-defined methods to measure acoustic effects of sinus surgery; the nasal tract was examined in isolation (without the vocal tract) and a standardized sine sweep for acoustical excitation was employed. Cadaveric situs as well as corresponding 3D casts provided stable anatomical conditions without any changes of sinonasal tract shape. By applying the minimally invasive maltodextrin mass occlusion we first could eliminate the sinus cavities from the acoustical system and then gradually include them into the acoustical landscape, one by one, in both pre- and postoperative conditions, respectively. Inclusion of a cavity into the resonatory system of the sinonasal tract generally led to an absorption of the sound energy at specific frequency. 
The frequency region, in which the sound absorption (represented by a marked dip in the transfer function) occurred, was shown to be dependent on the cavity volume.

Moreover, calculations of resonance frequencies expected for the given cavity dimensions, determined by volumetric measurements from the CT scan imaging, correlated significantly with experimentally measured dip frequencies in the cadaver as well as in the 3D replica, respectively. Despite the high spatial resolution of the axially acquired $\mathrm{CT}$ slices $(0.5 \mathrm{~mm})$, the exact dimensions as well as the complex shape of the narrow ostia region are difficult to delineate in the replica. Additionally, in the calculation via Helmholtz equation a tubular shape of the ostia was assumed. Thus, some differences between the obtained dip frequencies in the cadaver and the replica as well as in the calculated resonance frequencies could be expected.

The formalin-preservation of the human sinonasal tracts has led to hardening of the mucosal tissue as compared to an in vivo condition, fresh frozen or Thiel-fixation. However, the differences in the properties of the wall linings could not possibly affect the resonance frequencies. They can only affect the losses in the acoustic system and hence the depth of the dip in the transfer function.

Unexpectedly, in six cases, all in the preoperative condition, no dip war observed after removal of the ostium occlusion, Apart from the possibility of an incomplete removal of the occluding maltodextrin mass, the reason may have been that the uncinate process prevented excitation of the cavity in the case of the four middle meatus. The two sphenoidal ostia may have been too narrow to allow acoustical excitation.

Several publications investigating the acoustic properties of the nose experimentally or via mathematical calculation used Helmholtz resonators for modeling the paranasal cavities $(3,14,17)$. The present results show that the dip frequencies derived from the Helmholtz equation might provide a fairly good match of what was experimentally measured in the real human sinonasal anatomy.

We analyzed the effect of ostium occlusion removal on one side only. The CT scans showed a substantial asymmetry in cavity size between the left and right sides in the cadaver heads. As the cavity size strongly influences the resonance of the cavity, this asymmetry can be predicted to cause different dip frequencies between the left and right sides.

The obtained transfer functions were further analyzed with respect to changes in transmission characteristics following FESS procedures. The analysis revealed a systematic effect in terms of an increase of the dip frequency in the postoperative condition; in the cadaver as well as in the replica the dip frequencies increased by about $200-300 \mathrm{~Hz}$ as compared to the preoperative condition.

Endoscopically controlled widening of the maxillary and sphenoid ostia, established in the surgical routine for ventilation improvement of the sinus cavities in treatment for chronic rhinosinusitis with and without nasal polyps ${ }^{(27,29,30)}$, was observed to lead to significant increase in dip frequency. Extended intervention (frontosphenoethmoidectomy), typically reserved for pronounced sinonasal pathology and associated with comorbidities (cystic fibrosis, aspirin sensitivity) or revision procedures, showed additional increase of dip frequency.

Under ex vivo conditions, acoustic effect was detected of surgically widening the ostium of the maxillary and the sphenoid cavity. The effect, an increase of the associated dip frequency, was greater and significant only for the maxillary sinus. It would be relevant that the widening of maxillary ostia is typically more pronounced due to the anatomical conditions as well as to the clinical relevance of the mucosal pathology of the maxilloethmoidal region. Also, the axial length of the ostia is extremely small because of the thin lamella. With respect to the sphenoidal ostia the surgical widening is generally quite moderate. This may be the reason why the effect of surgery on the dip frequency failed to reach statistical significance.

Despite the common assumption that the frontal sinuses provide substantial resonatory effects on the voice, only one experimental study supporting this hypothesis could be identified in the literature. While subjects were phonating a nasal consonant, Dang et al. slided a flexible tube, attached to a probe microphone, along the floor of one side of the nasal cavity starting about $8 \mathrm{~cm}$ posteriorly, moving anteriorly towards the nostrils ${ }^{(14)}$. They observed acoustic alterations of dips in the high frequency region in 3 subjects and ascribed this to frontal sinus resonance. Also in the present work, dips were detected in the high frequency region, which could be explained by frontal sinus resonance, but only after completion of a frontosphenoethmoidectomy procedure with widening of frontal recess. Without surgical widening of the ostia, no acoustic effects of the frontal sinuses were observed in the transfer function in our examination.

It might further be mentioned that from an anatomical point of view and under normal conditions, i.e. without surgical intervention, the accessibility of the frontal sinuses and the ethmoidal cells is limited. The passages leading to these sinuses consist of the middle meatus region, the ethmoidal infundibulum, and the frontal recess, and they are typically so narrow that they would rather be inaccessible to acoustical excitation. In a retrospective study on multiplanar CT scan imaging in 641 patients, Leunig et al. found considerable diversity in paranasal sinus morphology, inter alia a prevalence of agger nasi cells located in the area of frontoethmoidal complex complicating the accessibility of the frontal sinus in $80 \%$ of the examined cases ${ }^{(31)}$. Moreover, Möller and associates showed that while a pulsating aerosol inhalation was found to deposit in the sphenoidal and maxillary sinuses, no such deposition was detected in the frontal sinuses ${ }^{(32)}$ confirming the severely limited accessibility of the frontal sinuses in 
unchanged ostia conditions.

Our investigation was focused on acoustic characteristics of the sinonasal tract in isolation. This favorably reduced complexity and facilitated theoretical understanding of findings, showing a reasonable quantitative agreement between theoretically calculated and experimentally measured resonances in cadavers and corresponding replicas. In this sense our investigation seems to have improved the understanding the acoustical effects of surgical interventions of the nasal tract. A relevant question, open for the future, concerns the consequences of endonasal surgery for the voice, as such surgery will affect not only the sinu-nasal resonance conditions - as demonstrated above - but also the control of the velopharyngeal opening in speech and singing.

\section{Conclusion}

Under normal conditions, the maxillary and sphenoidal sinus are accessible to sound excitation. Contrary to a common assumption, they absorb rather than amplify sound. The absorption is greatest at their resonance frequency, such that these sinuses produce dips in the frequency response of the nasal tract. The dip frequency was shown to be dependent from the volume of the sinus cavity. Using the Helmholtz equation the resonance frequencies of the paranasal sinus could be calculated from volumetric CT scan imaging, the results showing good accordance with the dip frequencies measured in the 3D replica as well as with data experimentally gained in real human sinonasal anatomy. Very narrow ostia impede sound access. Thus, no examples of sound absorption of the frontal sinuses could be documented, unless after surgical widening of the frontal recess. The FESS procedures lead to reproducible effects on the acoustical frequency response of the sinonasal tracts in terms of systematical increase of the dip frequencies.

\section{Authorship contribution}

$\mathrm{MH} / \mathrm{JS}$ conceptualized and designed the study. MH/SB/MS/AM/ TJ collected and assembled the data. Data analysis and interpretation were conducted by JS/MH, who also drafted the article. SB/MS/AM/TJ critically revised the article for intellectual content. All authors approved the final version of the article.

\section{Conflict of interest}

The authors describe no conflict of interest.

\section{References}

1. Wheeler $F$. The relation of the paranasal sinuses to the singing voice. Science 1930;Dec 19;72(1877):630.

2. Lindqvist-Gauffin J, Sundberg J. Acoustic properties of the nasal tract. Phonetica 1976;33(3):161-8

3. Maeda S. The role of the sinus cavities in the production of nasal vowels. Proc IEEE Int Conf Acoustics Speech and Signal Processing 1982;7:911-14.

4. Båvegård M, Fant G, Gauffin J, Liljencrants J. Vocal tract sweeptone data and mode simulations of vowels, laterals and nasals. STL- QPSR 1993;34:43-76.

5. Negus $V$. The function of the paranasal sinuses. AMA Arch Otolaryngol 1957;66(4):430-42.

6. Flottes I, Clere P, Rui R, Devilla F. la physiologie des sinus (Societe francaise d'OtoRhino-Laryngologie). Paris: libraire arnette: 1960. p. 359-60.

7. Schaeffer JP. The nose, the paranasal sinuses, nasolachrymal passageways and olfactory organ in man. Am J Med Sci 1920;160(1):122-128

8. Tepper G, Haas R, Schneider B, et al. Effects of sinus lifting on voice quality. A prospective study and risk assessment. Clin Oral Implants Res 2003;14(6):767-74

9. Ozbal Koc EA, Koc B, Ercan I, Kocak I, Tadihan E, Turgut S. Effects of septoplasty on speech and voice. J Voice 2014; May28(3):393.e11-5.

10. Liapi A, Hirani S, Rubin J. Changes in nasal resonance following septoplasty in adults: Acoustic and perceptual characteristics.
Logoped Phoniatr Vocol 2015 Apr 6:1-8.

11. Hosemann W, Göde U, Dunker JE, Eysholdt $U$. Influence of endoscopic sinus surgery on voice quality. Eur Arch Otorhinolaryngol 1998;255(10):499-503

12. Chen MY, Metson R. Effects of Sinus Surgery on Speech. Arch Otolaryngol Head Neck Surg 1997; 123: 845-852.

13. Fujimura $\mathrm{O}$, Lindqvist J. Sweep-tone measurements of vocal-tract characteristics. J Acoust Soc Am 1971 Feb:49(2):Suppl 2:541.

14. Dang J, Honda K, Suzuki H. Morphologica and acoustical analysis of the nasal and the paranasal cavities. J Acoust Soc Am 1994 Oct;96(4):2088-100.

15. Dang J, Honda K. Acoustic characteristics of the human paranasal sinuses derived from transmission characteristic measurement and morphological observation. J Acoust Soc Am 1996;100(5):3374-83.

16. Braun $T$, Rich $M$, Berghaus $A$, Kramer MF. Effects of oxymetazoline nasal spray on the nasal cycle assessed by long-term rhinoflowmetry. Rhinology 2012; 50(4):370-5.

17. Pruthi T, Espy-Wilson CY, Story BH Simulation and analysis of nasalized vowels based on magnetic resonance imaging data. J Acoust Soc Am 2007;121:3858-3873.

18. Maeda S. Acoustic Correlates of Vowe Nasalization: A Simulation Study. J Acoust Soc Am Suppl 1.1982;72, p 102

19. Koyama T. Experimental study on the resonance of paranasal sinus. J Otolaryngol Jpn 1966:69(6):1177-1191.

20. Behrman A, Shikowitz MJ, Dailey S. The effect of upper airway surgery on voice. Otolaryngol Head Neck Surg 2002
Jul;127(1):36-42

21. Soneghet $R$, Santos RP, Behlau $M$, Habermann W, Friedrich G, Stammberger $\mathrm{H}$. Nasalance changes after functional endoscopic sinus surgery. J Voice 2002 Sep;16(3):392-7.

22. Birkent $H$, Erol $U$, Ciyiltepe $M$, Eadie $T L$, Durmaz A, Tosun F. Relationship between nasal cavity volume changes and nasalance. J Laryngol Otol 2009 Apr;123(4):407-11.

23. Hong KH, Kwon SH, Jung SS. The assessment of nasality with a nasometer and sound spectrography in patients with nasal polyposis. Otolaryngol Head Neck Surg 1997 Oct;117(4):343-8.

24. Jiang RS, Huang HT. Changes in nasal resonance after functional endoscopic sinus surgery. Am J Rhinol 2006 JulAug;20(4):432-7.

25. Simmen D, Jones N. Manual of Endoscopic Sinus Surgery and its Extended Applications. Stuttgart: Thieme, 2005.

26. Havel M, Ertl L, Bauer D, Schuster M, Stelter $K$, Sundberg J. Resonator properties of paranasal sinuses: preliminary results of an anatomical study. Rhinology 2014;52(2):178-82.

27. Stammberger $\mathrm{H}$, Posawetz W. Functional endoscopic sinus surgery. Concept, indications and results of the Messerklinger technique. Eur Arch Otorhinolaryngol 1990;247(2):63-76.

28. Fokkens WJ, Lund VJ, Mullol J, et al. European Position Paper on Rhinosinusitis and Nasal Polyps 2012. Rhinol Suppl. 2012 Mar;(23):3 pp. 1-298

29. Bassiouni A, Chen PG, Wormald PJ. Mucosal remodeling and reversibility in chronic rhi- 
nosinusitis. Curr Opin Allergy Clin Immunol 2013 Feb;13(1):4-12.

30. Wormald PJ. Outcomes after endoscopic sinus surgery-important and valuable to everyday practice. Int Forum Allergy Rhino 2012 Nov;2(6):435-6.

31. Leunig A, Betz CS, Sommer B, Sommer F Anatomic variations of the sinuses; multiplanar CT-analysis in 641 patients. Laryngorhinootologie 2008;87:482-9.

32. Möller W, Schuschnig U, Celik G, Münzing W, Bartenstein P, Häussinger K, et al. Topical drug delivery in chronic rhinosinusitis patients before and after sinus surgery using pulsating aerosols. PLoS One 2013 Sep 11;8(9):e74991.
PD Dr. med. Miriam Havel

Dept. of Otorhinolaryngology

Head \& Neck Surgery

Section Phoniatrics

Munich University Hospital

Marchioninistr. 15

81377 Munich

Germany

Tel: +49-89-4400-73888

Fax: +49-89-4400-76869

E-mail: miriam.havel

@med.uni-muenchen.de

\section{ADVERTISEMENT}

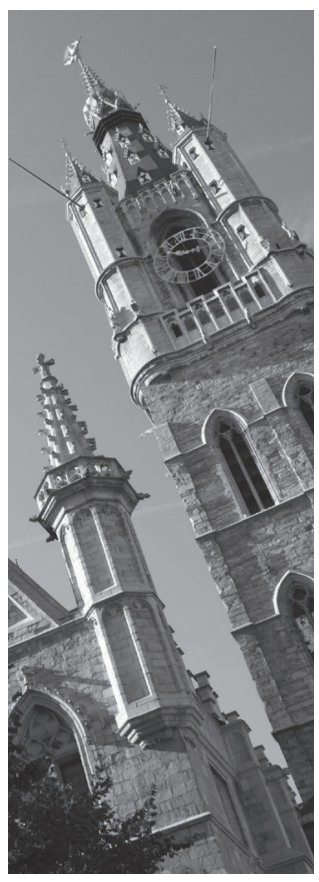

REGISTRATION FEE BEFORE JUNE $1^{\text {ST }} 2017$

ENT specialist

$1750 €$

ENT resident in training* $\quad 1350$

Lectures only

Accompanying person

$800 €$

*Reduced registration fee after receipt certificate Urits UNDER ERS AUSPICES

\section{9th International Course on \\ ENDOSCOPIC SURGERY \\ OF THE PARANASAL \\ SINUSES}

\&

\section{SKULL BASE}

Ghent (Belgium)

23-26 August 2017

\section{www.FESS-COURSE.be}

\section{THE COURSE FEATURES}

$12 \mathrm{hrs}$ fully equipped Hands-on Cadaver Head Workshop

- Two hands-on cadaver head workshops

- A full head/participant

- Endoscope, monitor and microdebrider/participant

- Cadaver head demonstrations of current techniques

- Surgery with navigation

Lectures, Round tables and Video-session

- Focused key-note presentations on endoscopic sinus surgery

and its extensions (DCR, skull base, tumours,,...$)$

- Video sessions

- Round tables, with interactive discussion with the faculty

members.

\section{EXTENDED INTERNATIONAL FACULTY}

Claus Bachert (Ghent, Belgium)

Manuel Bernal Sprekelsen (Barcelona, Spain)

Philippe Gevaert (Ghent, Belgium)

Werner Hosemann (Greifswald, Germany)

Bradley Marple (Dallas, USA)

Pär Stiärne (Stockholm, Sweden)

Thibaut Van Zele (Ghent, Belgium)

James Zinreich (Baltimore, USA) 